\title{
Keyhole Pattern with Superior-Medial Pedic in Large Breast Reduction
}

\author{
AHMED A. ZIDAN, M.D.; EHAB Z. AZZAM, M.D. and HOSSAM Y. EL-KAFRAWY, M.D. \\ The Department of General Surgery, Plastic Surgery Unit, Faculty of Medicine, Alexandria University, Egypt
}

\begin{abstract}
Background: Breast surgery is one of the most difficult fields of aesthetic surgery, because of the complexity of surgical procedures and the relative guidelines defining the aesthetically perfect breast.

The goal in reduction mammaplasty is to achieve a pleasing balance among size, shape, and projection of the breast with optimal sensation and appropriate blood supply for the nippleareola and breast. These goals are challenged by the amount of excess tissue and severely distorted breast landmarks in massive breast hypertrophy and severe breast ptosis.
\end{abstract}

The traditional treatment for massive breast hypertrophy or severe breast ptosis is breast amputation with free grafting of the nipple-areola or inverted $\mathrm{T}$, inferior pedicle used to have a relatively high number of complications and the disadvantage. On the other hand, relying on inverted T, superior-medial pedicle can avoid the disadvantage of the previse techniques and give reliable, stable and pleasant results in large reduction mammoplasties.

Patients and Methods: This is a prospective randomized study that included 20 healthy female patients suffering from breast hypertrophy and they are candidate for reduction mammoplasty by the proposed technique. Design of skin marking for superior-medial pedicle breast reduction technique was carried out with the standard Wise pattern. The amount of removed tissue was recorded, the aesthetic result and patient satisfaction was estimated for the results.

Results: This study includes 20 female patients of an age ranged between $24-45$ years old and a breast size (suprasternal notch to nipple distance) ranging from 33 to $42 \mathrm{~cm}$. The weight of removed tissues during surgery in our study was ranging from 1210 to 1970 . We found that $75 \%$ of our results are of excellent aesthetic results, $15 \%$ good and $10 \%$ fair with $70 \%$ of patients were satisfied, $20 \%$ of patients were somewhat satisfied/somewhat dissatisfied and $10 \%$ of patients were dissatisfied.

Conclusion: From this study we concluded that the keyhole pattern with superior medial pedicle was an efficient technique for excising more tissue in large breasts with pleasant aesthetic results.

Key Words: Keyhole - Breast reduction.

\section{INTRODUCTION}

Breast surgery is one of the most difficult fields of aesthetic surgery, because of the variety of surgical procedures and the relative guidelines defining the aesthetically perfect breast. The goals in reduction mammaplasty are to achieve a pleasing balance among size, shape, and projection of the breast with optimal sensation and appropriate blood supply for the nipple-areola and breast [1]. These goals are challenged by the amount of excess tissue and severely distorted breast landmarks in massive breast hypertrophy and severe breast ptosis [2]

The most common treatment for massive breast hypertrophy or severe breast ptosis is breast amputation with free grafting of the nipple-areola. The usual complications of this technique include; temporary or permanent loss of sensation, necrosis, hypopigmentation, and flatness of the nipple-areola [3].

Another traditional technique is the Inverted T/Keyhole pattern with inferior pedicle. Despite its popularity the inferior pedicle technique suffers some disadvantages that become more noticeable with large breast reductions [4].

In this study we utilize the Inverted T/Keyhole pattern with superior-medial pedicle believing its more efficient technique for large breast reduction with solution for most of the disadvantages of the previse techniques [5-7].

\section{PATIENTS AND METHODS}

This is a prospective randomized study that included 20 healthy female patients suffering from breast hypertrophy and they are candidate for reduction mammoplasty by the proposed technique. The patients were managed in Plastic and Reconstructive Surgery unit in Alexandria University Hospitals.

The breasts were marked for reduction in the upright position using the standard Wise pattern centered on the premarked meridian of the breast. The new nipple position was marked just below the inframammary fold by about $1-2 \mathrm{~cm}$ taking in consideration the marked skin recoil seen in large 
reduction. The new nipple position was planned in the range $22-25 \mathrm{~cm}$ from the sternal notch depending on the patient's stature and desired final breast size. Limit the horizontal limb laterally by the anterior axillary fold and medially $1-2 \mathrm{~cm}$ from the mid-line. Finally the pedicle was marked with width rang $8-10 \mathrm{~cm}$ and length not exceeding $15 \mathrm{~cm}$ Fig. (1).

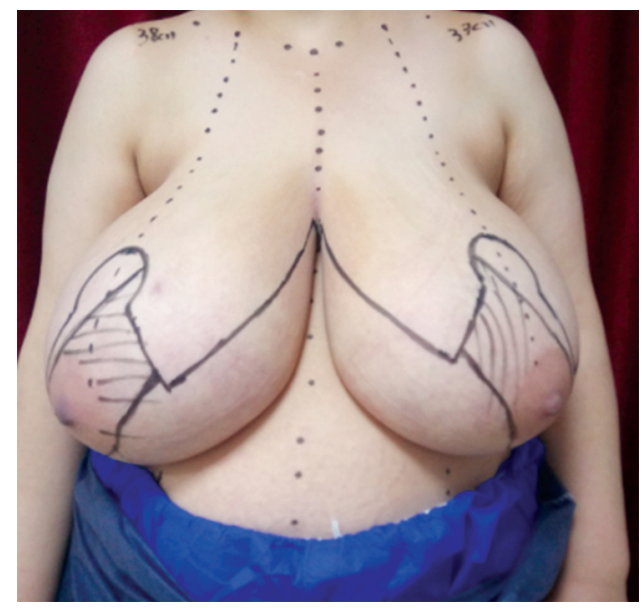

Fig. (1): Show the marking of keyhole pattern/superior pedicle breast reduction.

First the superiomedial pedicle was deepithelialized, the pedicle was incised on the under surface with the nipple-areola complex and a layer of parenchyma were elevated off the breast with a thickness $2-3 \mathrm{~cm}$ at the areola to approximately $4-$ $5 \mathrm{~cm}$ at the base.

The breast was split horizontally down to the pectoral fascia and the lower segment of the breast is removed in the form of a horizontal ellipse of skin and breast tissue. Care should be taken to avoid over resection the medial segment of the breast, while large resection usually take place in the lateral segment.

The vertical wedge of breast tissue was then excised while staying deep into the pedicle. Additional trimming and shaping of the medial and lateral segments of the remaining breast tissue could be performed.

In the occasional situation where there was concern about nipple-areola circulation, conversion to a nipple graft technique was an easy second option before the end of the procedure.

Standard breast photography (front, right and left views) was carried out pre-operative and postoperative for a minimum period of six months to assess the results of surgery as regards wound healing, scar quality, and breast shape.

\section{RESULTS}

The present study was conducted on twenty female patients with Breast Hypertrophy admitted to Plastic Surgery Unit at Alexandria Main University Hospital.

\section{I- Demographic data:}

- Age:

The study included 20 females. Their ages ranged from 24 years old to 45 years old. The mean age was 33.9 years (Table 1, Fig. 2).

Table (1): Distribution of the studied cases according to age (years) $(n=20)$.

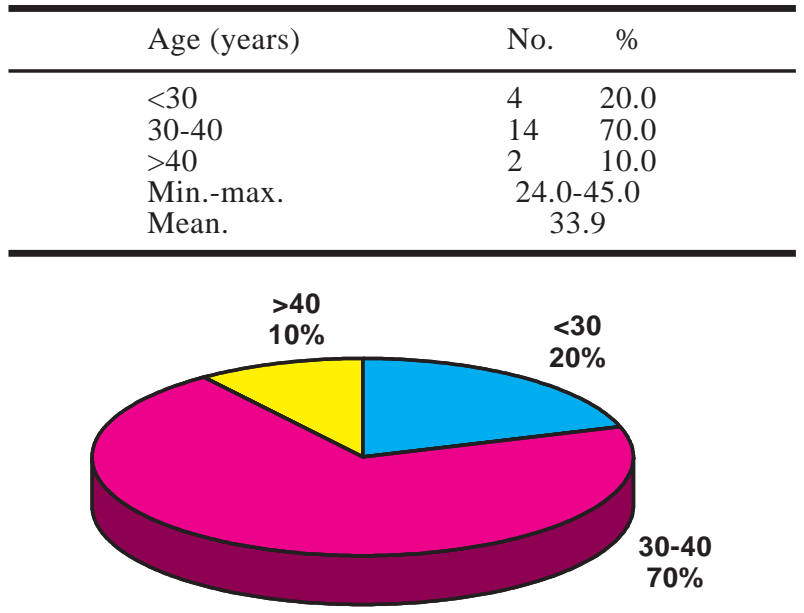

Fig. (2): Distribution of the studied cases according to age (years).

\section{- Suprasternal notch to nipple distance in $\mathrm{cm}$ :}

The breast size in our study was ranging from 33 to $42 \mathrm{~cm}$ with a mean of $(37.2 \pm 2.44) \mathrm{cm}$ in the right side and from 34 to $41 \mathrm{~cm}$ with a mean $(37.6 \pm$ $2.23) \mathrm{cm}$ in the left side (Table 2, Fig. 3).

Table (2): Descriptive analysis of the studied cases according to notch to NAC distance (CM).

\begin{tabular}{lll}
\hline $\begin{array}{l}\text { Notch to NAC } \\
\text { distance (CM) }\end{array}$ & Right & \multicolumn{1}{c}{ Left } \\
\hline Min.-max. & $37.0-42.0$ & $34.0-41.0$ \\
Mean \pm SD & $37.2 \pm 2.44$ & $37.6 \pm 2.23$ \\
Median & 37.00 & 37.50 \\
\hline
\end{tabular}

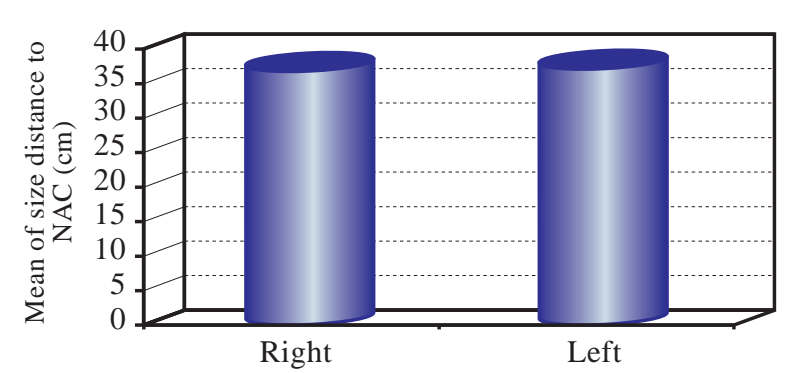

Fig. (3): Descriptive analysis of the studied cases according to notch to NAC distance $(\mathrm{CM})(\mathrm{n}=20)$. 


\section{- Weight of removed tissue:}

The weight of removed tissues during surgery in our study was ranging from 1210 to 1970 grams with a mean of $1531 \pm 224.91$ grams and median was 1560 grams in the right side and from 1240 to 1900 grams with a mean of $1568 \pm 200.53$ grams and median was 1615 grams in the left side (Table 3, Fig. 4).

Table (3): Descriptive analysis of the studied cases according to the weight of removed tissue $(n=20)$.

\begin{tabular}{lll}
\hline Removed tissue $(\mathrm{g})$ & \multicolumn{1}{c}{ Right } & \multicolumn{1}{c}{ Left } \\
\hline Min.-max. & $1210-1970$ & $1240-1900$ \\
Mean \pm SD & $1531 \pm 224.91$ & $1568 \pm 200.53$ \\
Median & 1560 & 1615 \\
\hline
\end{tabular}

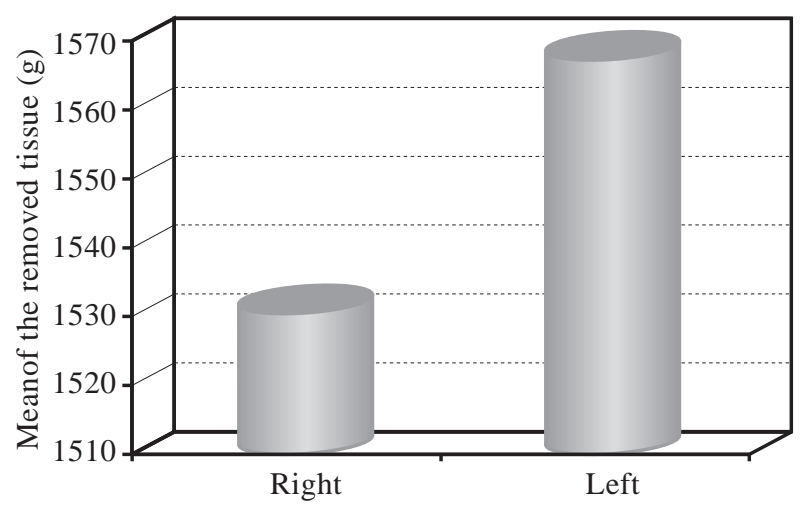

Fig. (4): Descriptive analysis of the studied cases according to removed tissue $(n=20)$.

\section{- Aesthetic results:}

We found that $75 \%$ of our results are of excellent aesthetic results, $15 \%$ good and $10 \%$ fair (Table 4, Fig. 5).

Table (4): Distribution of the studied cases according to aesthetic results $(n=20)$.

\begin{tabular}{lll}
\hline Patient satisfaction & No. & $\%$ \\
\hline Excellent & 15 & 75.0 \\
Good & 3 & 15.0 \\
Fair & 2 & 10.0 \\
\hline
\end{tabular}

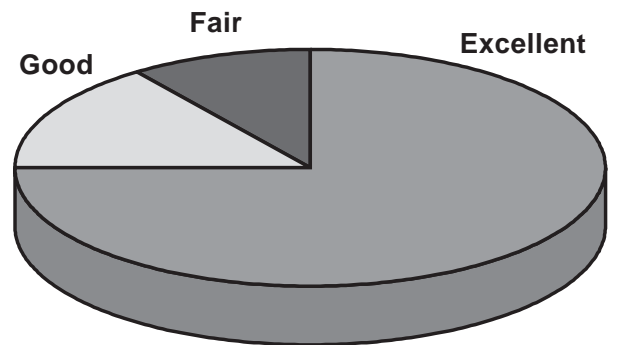

Fig. (5): Distribution of the studied cases according to patient satisfaction $(n=20)$.

\section{- Patient satisfaction:}

We found that $70 \%$ of patients were satisfied, $20 \%$ of patients were somewhat satisfied/somewhat dissatisfied and $10 \%$ of patients were dissatisfied (Table 5, Fig. 6).

Table (5): Distribution of the studied cases according to patient satisfaction $(n=20)$.

\begin{tabular}{llc}
\hline Patient satisfaction & No. & $\%$ \\
\hline Dissatisfied & 2 & 10.0 \\
Somewhat satisfied/somewhat dissatisfied & 4 & 20.0 \\
Satisfied & 14 & 70.0 \\
\hline
\end{tabular}

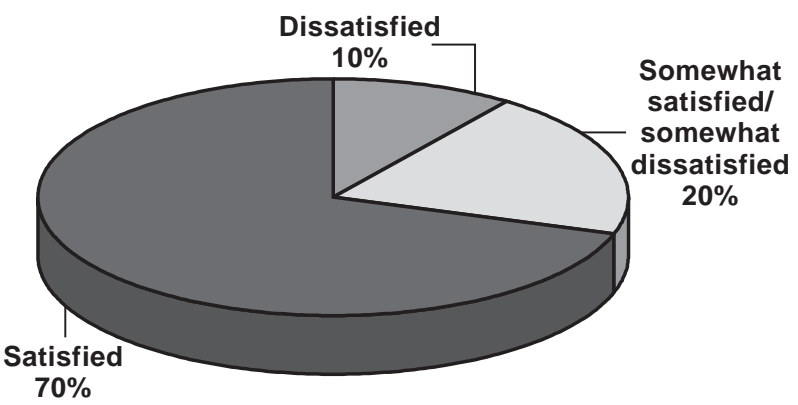

Fig. (6): Distribution of the studied cases according to patient satisfaction $(n=20)$.

\section{- Complications:}

A- Hematoma: Hematoma occurred in one case $(5 \%)$ in the left breast (Table 6).

B- Seroma: Seroma occurred in one case $(5 \%)$ in the left breast (Table 6).

C-Infection: There was no reported cases with infection (Table 6).

D- Wound healing problems: There were wound healing problems in 3 cases in our study at the T-junction (Table 6).

E- Partial Nipple loss: There was partial nipple loss in 3 cases $(15 \%) 1$ case with bilateral partial loss and 2 cases with unilateral partial loss (Table 6).

F- Fat necrosis: There was fat necrosis in 1 case (5\%) (Table 6).

Table (6): Recorded complications of the cases $(n=20)$.

\begin{tabular}{lccccc}
\hline & \multicolumn{2}{c}{ Right } & & \multicolumn{2}{c}{ Left } \\
\cline { 2 - 3 } \cline { 6 - 6 } & No. & $\%$ & & No. & $\%$ \\
\hline Haematoma & 0 & 0.0 & & 1 & 5.0 \\
Seroma & 0 & 0.0 & & 1 & 5.0 \\
Infection & 0 & 0.0 & & 0 & 0.0 \\
Wound healing problems & 1 & 5.0 & & 3 & 15.0 \\
Partial nipple loss & 1 & 5.0 & & 3 & 15.0 \\
Fat necrosis & 1 & 5.0 & & 0 & 0.0 \\
\hline
\end{tabular}




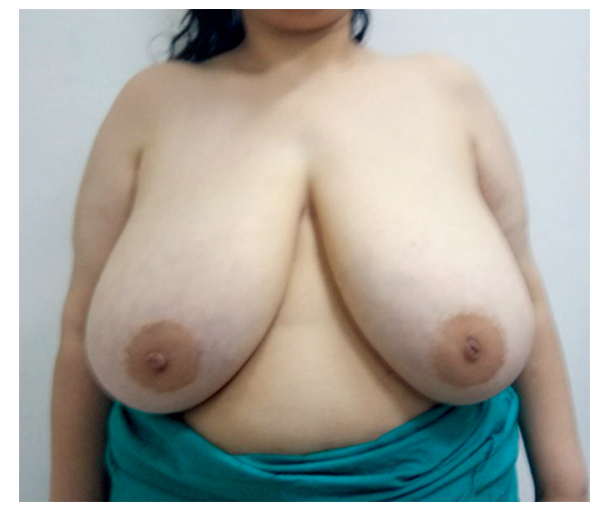

Cases presentation

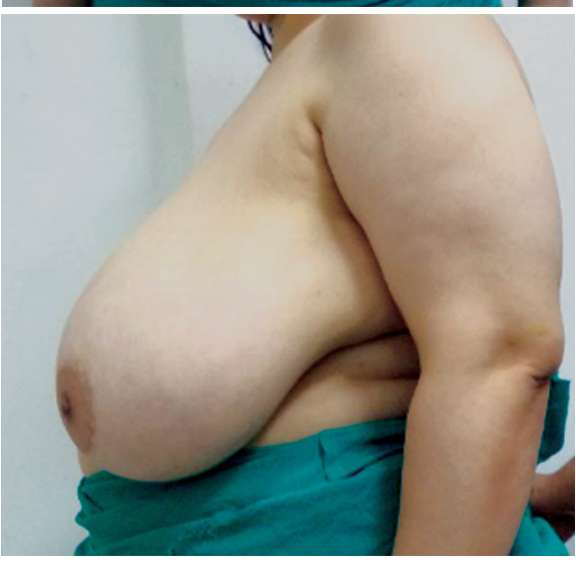

(A)
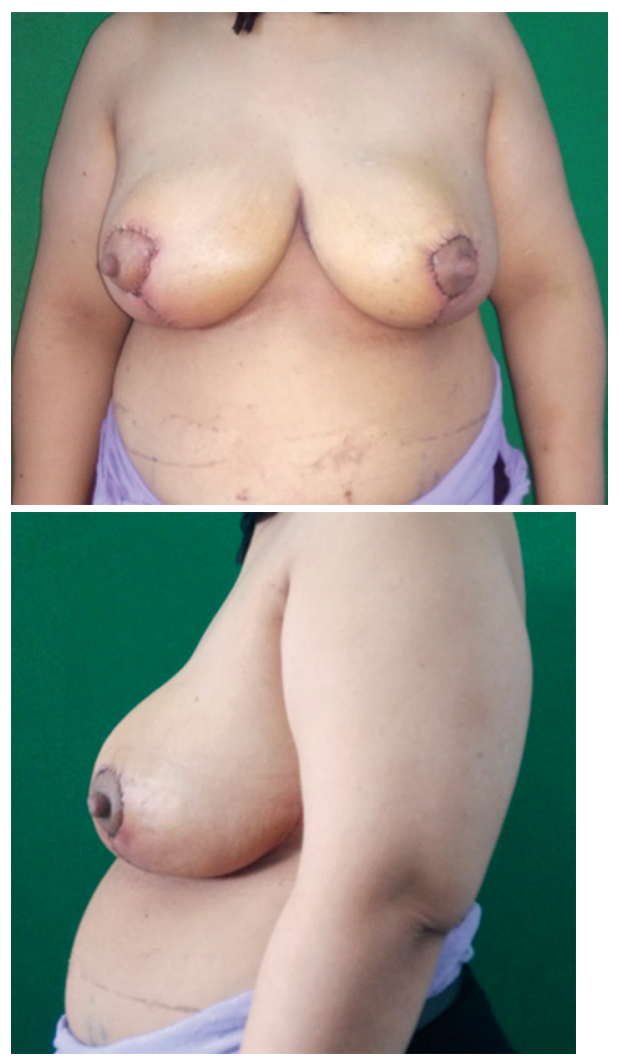

(B)

Fig. (7): 35-years (A) Pre-operative (anterior and lateral view) and (B) Post-operative (anterior and lateral view).

Case (2)
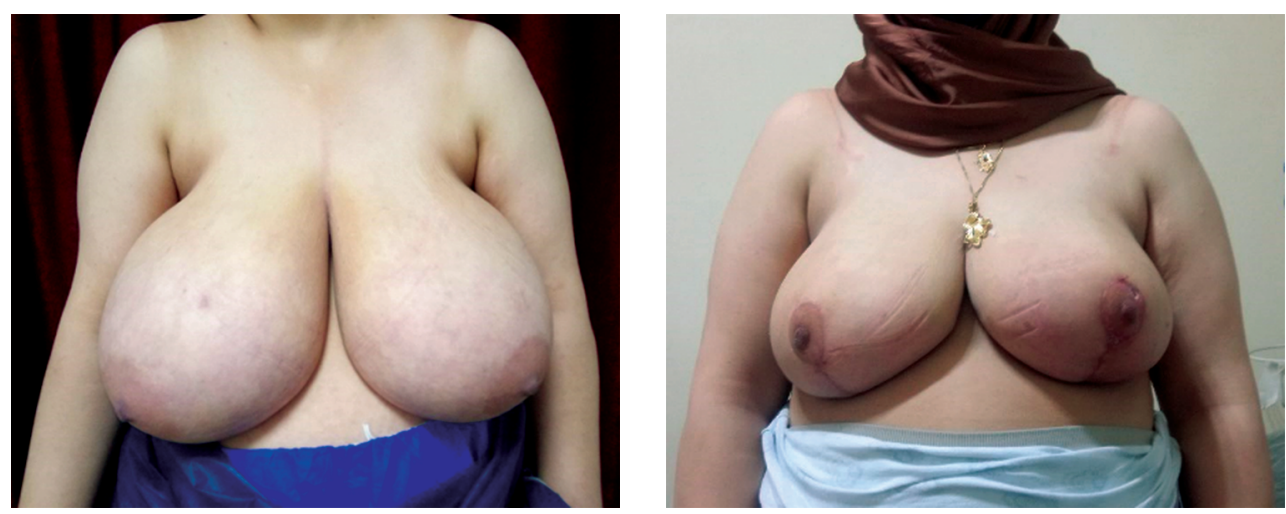

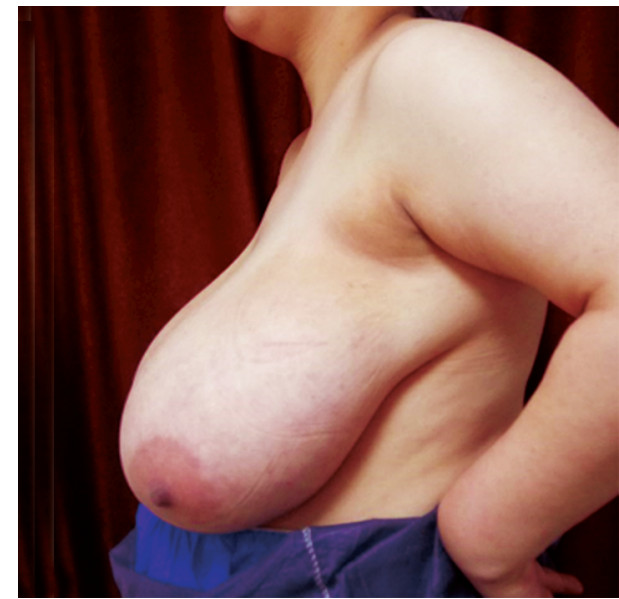

(A)

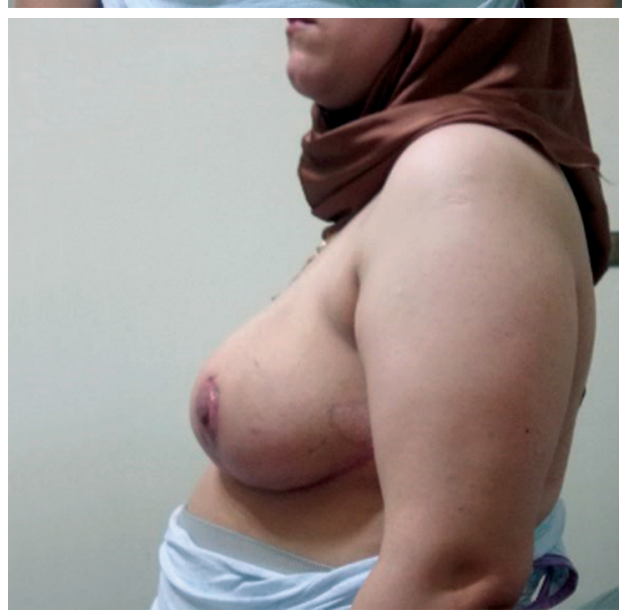

(B)

Fig. (8): 26-years (A) Pre-operative (anterior and lateral view) and (B) Post-operative (anterior and lateral view). 


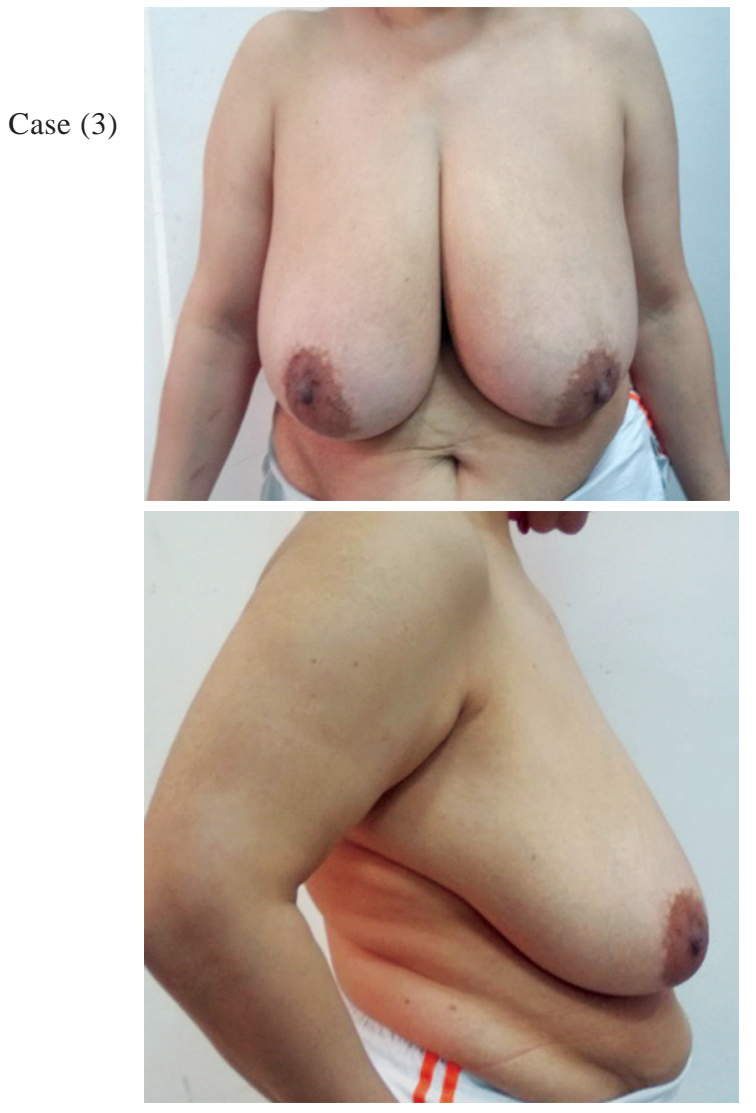

(A)

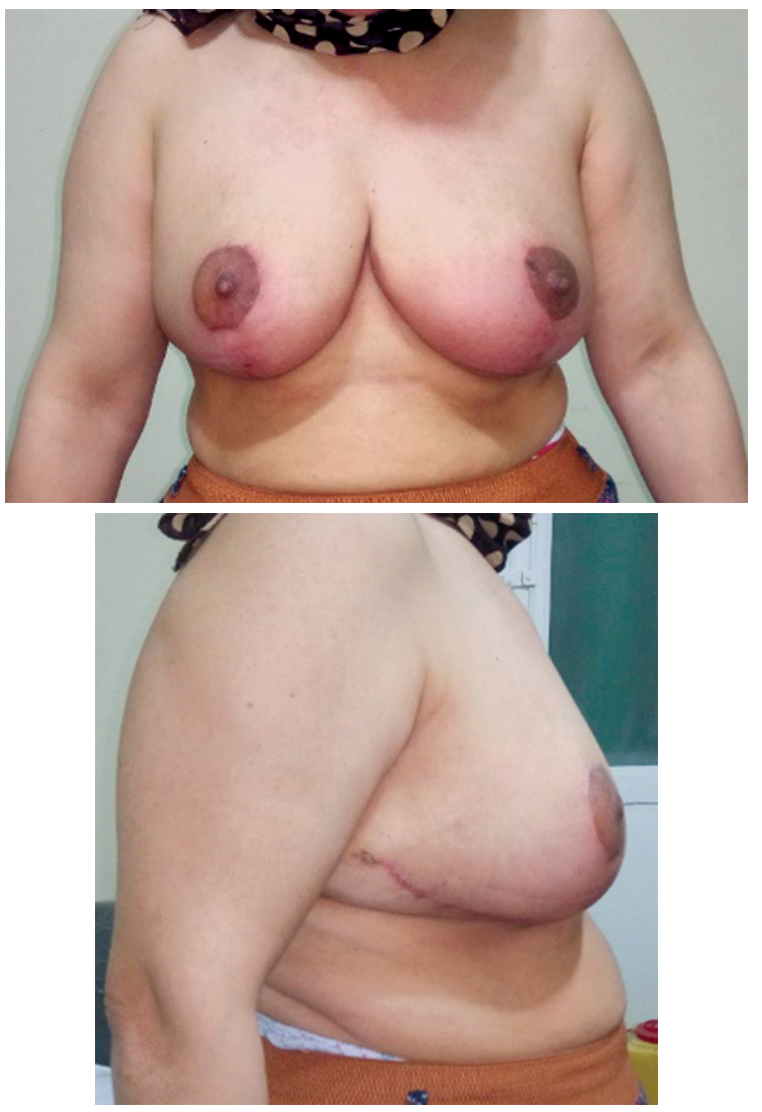

(B)

Fig. (9): 26-years (A) Pre-operative (anterior and lateral view) and (B) Post-operative (anterior and lateral view).

\section{DISCUSSION}

Large breast hypertrophy is a frequent condition in women population. It may occur due to excessive development of breast tissue during puberty, secondary to weight gain or as a result of naturally occurring fat deposition of breast tissue that takes place with aging.

Treatment of breast hypertrophy is strictly surgical, through breast reduction surgery which have the main objectives of reducing the amount of breast tissue, correction of breast shape, cranially reposition of the nipple-areola complex to a more aesthetic position. These objectives are meet by great challenges in large breast reduction that implies thorough choice of the technique used for this reduction surgery.

The commonly utilized technique of breast amputation with free grafting of the nipple-areola used to cause temporary or permanent loss of sensation, necrosis, hypopigmentation, and flatness of the nipple-areola complex [3].

The more popular inverted T/Keyhole pattern with inferior pedicle technique suffers from a number of "mechanical" disadvantages. First, the inferior pedicle is inferior and thus the excess tissue that is inferiorly situated cannot all be excised. More importantly, as the inferior pedicle lies under the point of maximum tension of the adjacent skin flaps, breakdown at the $\mathrm{T}$ is not uncommon. In fact, the more bulk in the pedicle, the more tension is applied to the T junction, with subsequent skin (and fat) necrosis. Disadvantages also includes extensive dissection of breast tissue, long operating times, and a tendency to "bottom out" over time [8,9].

The Wise keyhole pattern still remains the most common skin incision used in large reduction mammaplasty. It allows for flexibility in the method of nipple/areola transposition and gives a predictable result.

In our technique with the superior-medial pedicle the superficial mammary tissue of the upper pole has a rich intradermal blood supply created by the superficiality of the anterior perforating branches of the internal mammary artery [10].

The superior-medial pedicle technique had advantages including simplicity, easy reproducibil- 
ity, and shorter operating times. Unlike inferior pedicle it permits the excess tissue situated inferiorly to be excised without impedance, which involved removal of the lower segment of the breast together with a vertical wedge of central breast tissue, which when closed created the desired projection and shape.

Superiomedial pedicle techniques have also provided a safety transposition of the nipple-areola in reduction mammaplasty for large breast hypertrophy preserving normal areola skin texture and nipple appearance. Additionally, the pedicle is rotated from an inferior position to a superior position tissue is redistributed into a more appropriate location unlike inferior pedicle it permits the excess tissue situated inferiorly to be excised without impedance and so a more efficient technique for excising more tissue in large breasts with pleasant aesthetic results.

\section{REFERENCES}

1- Lickstein L.H. and Shestak K.C.: The conceptual evolution of modern reduction mammaplasty. Oper. Tech. Plast. Reconstr. Surg., 6 (2): 88-96, 1999.

2- Gabka C. and Bohmert H.: Introduction in Aesthetic and Plastic Surgery of the Breast. In: Plastic and reconstructive surgery of the breast, $2^{\text {nd }}$ edition, Thieme. Stuttgart \& New York, (1): 14, 2009.
3- Graf R. and Biggs T.M.: In search of a better shape in mastopexy and reduction mammoplasty. Plast. Reconstr. Surg., 110 (1): 309-17, 2002.

4- O'Grady K.F., Thoma A. and Dal Cin A.: A comparison of complication rates in large and small inferior pedicle reduction mammaplasty. Plast. Reconstr. Surg., 115 (3): 736-42, 2005.

5- Zhu V., Lentz R., Sturrock T., et al.: Three-dimensional analysis of postoperative volumetric distribution in reduction mammoplasty using a superomedial pedicle versus an inferior pedicle. Plast. Reconstr. Surg., 135 (4): 1258, 2015.

6- Finger R., Vasquez B., Drew G.S. and Given K.S.: Superomedial pedicle technique of reduction mammoplasty. Plast. Reconstr. Surg., 83: 471-80, 1989.

7- Davison S.P., Mesbahi A.N., Ducic I., et al.: The versatility of the superomedial pedicle with various skin reduction patterns. Plast. Reconstr. Surg., 120: 1466-76, 2007.

8- Erdogan B., Ayhan M., Deren O. and Tuncel A.: Importance of Pedicle Length in Inferior Pedicle Technique and LongTerm Outcome of Areola-to-Fold Distance. Aesth. Plast. Surg., 26: 436-43, 2002.

9- Abdelaal M.M. and Aboelatta Y.A.: Dermo-Fascial Suspension for Better Contouring and Long Lasting Results in Reduction Mammoplasty. Aesth. Plast. Surg., 39: 7883, 2014.

10- Spear S.L. and Howard M.A.: Evaluation of the vertical reduction mammoplasty. Plast. Reconstr. Surg., 112: 85568, 2003. 